\title{
Asymptotic properties of Markov operators defined by Volterra type integrals
}

\author{
by Karol Baron and Andrzej Lasota (Katowice)
}

\begin{abstract}
New sufficient conditions for asymptotic stability of Markov operators are given. These criteria are applied to a class of Volterra type integral operators with advanced argument.
\end{abstract}

Introduction. We shall study asymptotic properties of the iterates $\left(P^{n}\right)$ of the operator

$$
P f(x)=\int_{0}^{\lambda(x)} K(x, y) f(y) d y
$$

where

$$
K(x, y)=-\frac{\partial}{\partial x} H(Q(\lambda(x))-Q(y))
$$

and $Q, \lambda,-H$ are given nonnegative and nondecreasing functions defined on the half line $\mathbb{R}_{+}=[0, \infty)$. The precise assumptions concerning the kernel $K$ will be formulated in Section 2 .

Operators of the form (0.1), (0.2) appear in mathematical models of the cell cycle [5], [10], [11], [12] and in a model of the electrical activity of neurons [7].

In the special case when $H(x)=e^{-x}$, a sufficient condition for asymptotic stability of the sequence $\left(P^{n}\right)$ was recently given in [2]. It has the form

$$
\liminf _{x \rightarrow \infty}(Q(\lambda(x))-Q(x))>1 .
$$

In the general situation, with arbitrary $H$, condition (0.3) was replaced in [7] by

1991 Mathematics Subject Classification: Primary 47B38, 47B65, 47G10; Secondary 45D05, 60J05.

Key words and phrases: Markov operator, integral Markov operator, stationary density, asymptotic stability, sweeping. 


$$
\liminf _{x \rightarrow \infty} \frac{Q(\lambda(x))}{Q(x)}>1 .
$$

Since $Q(x)$ and $\lambda(x)$ converge to $+\infty$ as $x \rightarrow+\infty$, inequality (0.4) is much more restrictive than (0.3). In particular, $(0.4)$ is not satisfied in some cases important for applications. The purpose of the present paper is to formulate a sufficient condition of the form (0.3) for asymptotic stability of $\left(P^{n}\right)$ valid for a large class of functions.

The organization of the paper is as follows. Section 1 contains some auxiliary definitions and theorems from the theory of Markov operators. Our results in this area are based on special properties of integral and Harris operators [1]. In particular, our Theorem 1.2 extends a recent result of J. Malczak [8]. In Section 2 we discuss the asymptotic properties of the iterates of the operator $P$ given by formulas $(0.1),(0.2)$.

1. Markov operators. Let $(X, \mathcal{A}, \mu)$ be a $\sigma$-finite measure space. Denote by $D=D(X, \mathcal{A}, \mu)$ the subset of $L^{1}=L^{1}(X, \mathcal{A}, \mu)$ which contains all (normalized) densities, i.e.

$$
D=\left\{f \in L^{1}: f \geq 0,\|f\|=1\right\}
$$

where $\|\cdot\|$ stands for the norm in $L^{1}$. A linear mapping $P: L^{1} \rightarrow L^{1}$ is called a Markov operator if $P(D) \subset D$.

Let a Markov operator $P$ be given. A density $f$ is called stationary (or invariant) if $P f=f$. The operator $P$ is called asymptotically stable if there is a density $f_{*}$ such that

$$
\lim _{n \rightarrow \infty}\left\|P^{n} f-f_{*}\right\|=0 \quad \text { for } f \in D .
$$

Of course, a density $f_{*}$ satisfying condition (1.1) is necessarily stationary and unique.

In order to present a simple criterion for the existence of a stationary density we must recall the notion of Banach limits [4]. A Banach limit $L$ is a linear functional defined on the space $l^{\infty}$ of bounded sequences $\left(a_{n}\right)=$ $\left(a_{1}, a_{2}, \ldots\right)$ of real numbers which satisfies the following conditions:

(i) $L\left(a_{n}\right) \geq 0$ if $a_{i} \geq 0(i=1,2, \ldots)$,

(ii) $L\left(a_{1}, a_{2}, \ldots\right)=L\left(a_{2}, a_{3}, \ldots\right)$,

(iii) $L(1,1, \ldots)=1$.

If $\left(a_{n}\right)$ is convergent then $L\left(a_{n}\right)=\lim _{n \rightarrow \infty} a_{n}$, and if $\limsup a_{n} \leq c$ then $L\left(a_{n}\right) \leq c$.

Theorem 1.1. Let $P: L^{1}(X, \mathcal{A}, \mu) \rightarrow L^{1}(X, \mathcal{A}, \mu)$ be a Markov operator and $L$ a Banach limit. Assume that there exists a set $A \in \mathcal{A}, \mu(A)<\infty$, a 
number $\delta>0$ and a density $f$ such that

$$
L\left(\int_{(X \backslash A) \cup E} P^{n} f d \mu\right)<1 \quad \text { for } E \subset A \text { with } \mu(E)<\delta .
$$

Then $P$ admits a stationary density.

The proof of this result was given by J. Socała [9]. It should be noted, however, that in Socała's statement a stronger form of condition (1.2) was used. Namely, the functional $L$ was replaced by limsup. The above formulation was proposed by T. Komorowski and J. Tyrcha [3].

Now consider an operator $P$ of the form

$$
P f(x)=\int_{X} k(x, y) f(y) d \mu(y)
$$

where $k: X \times X \rightarrow \mathbb{R}$ is a stochastic kernel, i.e. $k$ is jointly measurable on $X \times X$ and satisfies

$$
\begin{aligned}
& k(x, y) \geq 0 \quad \text { for }(x, y) \in X \times X \\
& \int_{X} k(x, y) d \mu(x)=1 \quad \text { for } y \in X .
\end{aligned}
$$

From (1.4) it follows immediately that $P$ is a Markov operator; it is called an integral Markov operator.

For integral Markov operators the existence of an invariant density and a simple transitivity condition imply asymptotic stability. To formulate this criterion precisely recall that in the theory of Markov operators the support of an $f \in L^{1}(X, \mathcal{A}, \mu)$ is defined up to a set of measure zero by the formula

$$
\operatorname{supp} f=\{x \in X: f(x) \neq 0\} \text {. }
$$

We say that a Markov operator $P$ overlaps supports if for every $f, g \in D$ there is a positive integer $n_{0}=n_{0}(f, g)$ such that

$$
\mu\left(\operatorname{supp} P^{n_{0}} f \cap \operatorname{supp} P^{n_{0}} g\right)>0 .
$$

Observe that condition (1.5) implies that

$$
\mu\left(\operatorname{supp} P^{n} f \cap \operatorname{supp} P^{n} g\right)>0 \quad \text { for } n \geq n_{0}(f, g) .
$$

In fact,

$$
\operatorname{supp} P^{n} f \cap \operatorname{supp} P^{n} g \supset \operatorname{supp} P^{n-n_{0}}\left(\min \left\{P^{n_{0}} f, P^{n_{0}} g\right\}\right) .
$$

THEOREM 1.2. An integral Markov operator which overlaps supports and has a stationary density $f_{*}>0$ a.e. is asymptotically stable.

Proof. Define a new measure space $(X, \mathcal{A}, \bar{\mu})$ with $d \bar{\mu}=f_{*} d \mu$ and consider the operator

$$
\bar{P} f=\left(1 / f_{*}\right) P\left(f \cdot f_{*}\right) .
$$


Observe that for every $f \in L^{1}(\bar{\mu})$ the product $f \cdot f_{*}$ belongs to $L^{1}(\mu)$. It is evident that $\bar{P}$ is an integral operator on $L^{1}(\bar{\mu})$ and that

$$
\bar{P} 1_{X}=1_{X} .
$$

(Here and in the sequel $1_{E}$ denotes the characteristic function of the subset $E$ of $X$.) Now we are going to use a well known decomposition property of integral Markov operators satisfying $\bar{P} 1_{X} \leq 1_{X}$ (see [1], Ch. VIII). The space $X$ may be written in the form

$$
X=X_{1} \cup X_{2}, \quad X_{1}=\bigcup_{i} W_{i}
$$

where the family $\left\{W_{i}\right\}$ is at most countable. The sets $X_{1}, X_{2}$ and $W_{i}$ are measurable, disjoint $\left(X_{1} \cap X_{2}=\emptyset, W_{i} \cap W_{j}=\emptyset\right.$ for $\left.i \neq j\right)$ and have the following properties:

(i) For every $f \in L^{1}(\bar{\mu})$ with supp $f \subset X_{2}$ and for every $g \in L^{\infty}(\bar{\mu})$,

$$
\lim _{n \rightarrow \infty} \int_{X} g \cdot \bar{P}^{n} f d \bar{\mu}=0 .
$$

(ii) For every $i$ there is a $j$ such that $\bar{P} 1_{W_{i}}=1_{W_{j}}$.

(iii) Every set $W_{i}$ is either cyclic or wandering. In the first case $\bar{P}^{k} 1_{W_{i}}=$ $1_{W_{i}}$ for a positive integer $k$; in the second, all sets $W_{i n}(n=0,1, \ldots)$ defined by $1_{W_{i n}}=\bar{P}^{n} 1_{W_{i}}$ are distinct and hence disjoint.

(iv) For every cyclic $W_{i}$ with period $k$ and for every $f \in L^{1}(\bar{\mu})$ vanishing outside $W_{i}$,

$$
\lim _{n \rightarrow \infty}\left\|\bar{P}^{n k} f-\left(\int_{W_{i}} f d \bar{\mu} / \bar{\mu}\left(W_{i}\right)\right) 1_{W_{i n}}\right\|_{L^{1}(\bar{\mu})}=0 .
$$

We shall show that in our case the decomposition formula (1.8) reduces to $X=W_{1}$. In fact, $\bar{\mu}\left(X_{2}\right) \leq \bar{\mu}(X)=1$ and we may take $f=1_{X_{2}}, g=1_{X}$ in (1.9). Since $\bar{P}$ preserves the integral with respect to $\bar{\mu}$ this gives $\bar{\mu}\left(X_{2}\right)=0$. Assume that $W_{i}$ is wandering. Then

$$
\begin{aligned}
\operatorname{supp} P^{n}\left(f_{*} \cdot 1_{W_{i}}\right) \cap \operatorname{supp} P^{n}\left(f_{*} \cdot 1_{W_{i 1}}\right) & =\operatorname{supp} \bar{P}^{n} 1_{W_{i}} \cap \operatorname{supp} \bar{P}^{n} 1_{W_{i 1}} \\
& =W_{i n} \cap W_{i, n+1}=\emptyset
\end{aligned}
$$

for every $n$, which contradicts (1.5) and shows that there are no wandering sets. Assume now that $W_{i}$ is cyclic with period $k \geq 2$. Then, as previously,

$$
\begin{aligned}
\operatorname{supp} P^{k n}\left(f_{*} \cdot 1_{W_{i}}\right) \cap \operatorname{supp} P^{k n}\left(f_{*} \cdot 1_{W_{i 1}}\right) & =W_{i, k n} \cap W_{i, k n+1} \\
& =W_{i} \cap W_{i 1}=\emptyset
\end{aligned}
$$

for every $n$. Consequently, each $W_{i}$ is cyclic with period $k=1$. Assume that there are two such sets, say $W_{1}$ and $W_{2}$. Then

$$
\operatorname{supp} P^{n}\left(f_{*} \cdot 1_{W_{1}}\right) \cap \operatorname{supp} P^{n}\left(f_{*} \cdot 1_{W_{2}}\right)=W_{1} \cap W_{2}=\emptyset
$$


for every $n$, which again contradicts (1.5). Thus there is exactly one cyclic set with cycle length $k=1$. We denote this set by $W_{1}$. According to (1.10) with $\bar{\mu}\left(W_{1}\right)=\bar{\mu}(X)=1, k=1$, we obtain

$$
\lim _{n \rightarrow \infty}\left\|\bar{P}^{n} f-\left(\int_{X} f d \bar{\mu}\right) 1_{X}\right\|_{L^{1}(\bar{\mu})}=0
$$

for every $f \in L^{1}(\bar{\mu})$. Evidently, for every $f \in D(\mu)$ we have $f / f_{*} \in L^{1}(\bar{\mu})$ and

$$
\begin{aligned}
\left\|P^{n} f-f_{*}\right\|_{L^{1}(\mu)} & =\left\|f_{*} \bar{P}^{n}\left(f / f_{*}\right)-f_{*} \int_{X}\left(f / f_{*}\right) d \bar{\mu}\right\|_{L^{1}(\mu)} \\
& =\left\|\bar{P}^{n}\left(f / f_{*}\right)-\left(\int_{X}\left(f / f_{*}\right) d \bar{\mu}\right) 1_{X}\right\|_{L^{1}(\bar{\mu})} .
\end{aligned}
$$

From this and (1.11) we get (1.1).

Corollary 1.1. Let $P: L^{1}(X, \mathcal{A}, \mu) \rightarrow L^{1}(X, \mathcal{A}, \mu)$ be an integral Markov operator which has a positive stationary density $f_{*}\left(f_{*}>0\right.$ a.e. $)$. Assume, moreover, that there exists a set $A \in \mathcal{A}, \mu(A)>0$, with the following property. For every $f \in D$ there is a positive integer $n_{0}=n_{0}(f)$ such that

$$
P^{n_{0}} f(x)>0 \quad \text { for a.e. } x \in A \text {. }
$$

Then $P$ is asymptotically stable.

Theorems 1.1 and 1.2 do not match well. In fact, the invariant density existing by Theorem 1.1 need not be positive on the whole space $X$, which is an important assumption in Theorem 1.2. This situation may be improved by studying $P$ restricted to the support of the invariant density.

Let a Markov operator $P: L^{1}(X, \mathcal{A}, \mu) \rightarrow L^{1}(X, \mathcal{A}, \mu)$ be given. It is well known that for all nonnegative $f, f_{*} \in L^{1}(X)$ the inclusion supp $f \subset \operatorname{supp} f_{*}$ implies $\operatorname{supp} P f \subset \operatorname{supp} P f_{*}$. In particular, if $f_{*}=P f_{*}$ and $\operatorname{supp} f_{*}=C$ then

$$
\text { supp } f \subset C \text { implies } \operatorname{supp} P f \subset C \text {. }
$$

This property allows us to consider $P$ on the space $L^{1}(C)$ of all functions from $L^{1}(X)$ with supports contained in $C$. We will denote $P$ restricted to $L^{1}(C)$ by $P_{C}$.

TheOREM 1.3. Let $P: L^{1}(X, \mathcal{A}, \mu) \rightarrow L^{1}(X, \mathcal{A}, \mu)$ be a Markov operator having an invariant density $f_{*}$. Assume that the operator $P_{C}$ with $C=$ $\operatorname{supp} f_{*}$ is asymptotically stable. Assume, moreover, that there is a $\delta>0$ such that

$$
\sup _{n} \int_{C} P^{n} f d \mu \geq \delta \quad \text { for } f \in D(X) \text {. }
$$

Then $P: L^{1}(X) \rightarrow L^{1}(X)$ is also asymptotically stable. 
Proof. According to the lower bound function theorem (see [6], Ch. 5) in order to prove (1.1) it is sufficient to find a nonnegative $h \in L^{1}(X)$, $\|h\|>0$, such that

$$
\lim _{n \rightarrow \infty}\left\|\left(P^{n} f-h\right)^{-}\right\|=0 \quad \text { for } f \in D(X)
$$

where $\|\cdot\|$ stands for the norm in $L^{1}(X)$. Define $h=\frac{1}{2} \delta f_{*}$ and fix an $f \in D(X)$. According to (1.12) there is an integer $m$ such that

$$
\eta:=\int_{C} P^{m} f d \mu \geq \frac{1}{2} \delta .
$$

For $n \geq m$ we have

$$
P^{n} f=P^{n-m}\left(1_{X \backslash C} P^{m} f\right)+P_{C}^{n-m}\left(1_{C} P^{m} f\right) .
$$

Since $P_{C}$ is asymptotically stable with invariant density $f_{*}$ we also have

$$
\lim _{n \rightarrow \infty}\left\|P_{C}^{n-m}\left(1_{C} P^{m} f\right)-\eta f_{*}\right\|=0 .
$$

From (1.14) and the inequality $h \leq \eta f_{*}$ it follows that

$$
\left\|\left(P^{n} f-h\right)^{-}\right\| \leq\left\|P_{C}^{n-m}\left(1_{C} P^{m} f\right)-\eta f_{*}\right\|
$$

for $n \geq m$. This and (1.15) imply (1.13).

Using Theorems 1.2 and 1.3 it is easy to derive the following

Corollary 1.2. Let $P: L^{1}(X, \mathcal{A}, \mu) \rightarrow L^{1}(X, \mathcal{A}, \mu)$ be an integral Markov operator which overlaps supports and has an invariant density $f_{*}$. Set $C=\operatorname{supp} f_{*}$. If there is a $\delta>0$ such that (1.12) is satisfied, then $P$ is asymptotically stable.

Pr o of. According to Theorem 1.3 it is enough to prove that the operator $P_{C}$ is asymptotically stable. Evidently,

$$
P_{C} f(x)=\int_{C} k(x, y) f(y) d \mu(y)
$$

for every $f \in L^{1}(C)$ and

$$
\begin{aligned}
0 & =\int_{C} f_{*}(y) d \mu(y)-\int_{C} P_{C} f_{*}(x) d \mu(x) \\
& =\int_{C}\left(1-\int_{C} k(x, y) d \mu(x)\right) f_{*}(y) d \mu(y),
\end{aligned}
$$

whence

$$
\int_{C} k(x, y) d \mu(x)=1 \quad \text { for } \text { a.e. } y \in C \text {. }
$$

This shows that $P_{C}$ is an integral Markov operator. Thus we can apply Theorem 1.2 to $P_{C}$ and its asymptotical stability follows. 
2. Volterra operators. In this section we shall consider the integral operator $P$ defined by $(0.1)$ and (0.2) under the following general assumptions (K1) and (K2):

$(\mathrm{K} 1) H:[0, \infty) \rightarrow[0, \infty)$ is nonincreasing, absolutely continuous and

$$
H(0)=1, \quad \lim _{x \rightarrow \infty} H(x)=0 .
$$

$(\mathrm{K} 2) Q:[0, \infty) \rightarrow[0, \infty)$ and $\lambda:[0, \infty) \rightarrow[0, \infty)$ are nondecreasing, absolutely continuous and

$$
Q(0)=\lambda(0)=0, \quad \lim _{x \rightarrow \infty} Q(x)=\lim _{x \rightarrow \infty} \lambda(x)=\infty .
$$

The above conditions (K1) and (K2) are assumed in the whole of this section and will not be repeated in the statements of the theorems. Moreover, all measure-theoretic notions refer to the standard Lebesgue measure $m$ on $[0, \infty)$.

We start with the following lemma from [7].

Lemma 2.1. If $W:[0, \infty) \rightarrow[0, \infty)$ is measurable and $f \in D$, then

$$
\int_{0}^{\infty} W(Q(\lambda(x))) \operatorname{Pf}(x) d x=\int_{0}^{\infty} f(y) d y \int_{0}^{\infty} W(x+Q(y)) h(x) d x
$$

where

$$
h(x)=-H^{\prime}(x) .
$$

Using Theorem 1.1 we prove the following theorem concerning the existence of a stationary density for $P$.

THEOREM 2.1. If there exists an $\alpha \in(0,1]$ such that

$$
\int_{0}^{\infty} x^{\alpha} h(x) d x<\liminf _{x \rightarrow \infty}\left((Q(\lambda(x)))^{\alpha}-Q(x)^{\alpha}\right),
$$

then the operator $P$ given by formulas (0.1), (0.2) has a stationary density.

Proof. Evidently, $P$ is an integral Markov operator defined on $L^{1}([0, \infty))$. Define

$$
\sigma=\int_{0}^{\infty} x^{\alpha} h(x) d x
$$

Using (2.2) we can find positive numbers $\varepsilon, \varrho$ and $x_{0}$ such that

$$
\sigma+\varepsilon<\varrho<(Q(\lambda(x)))^{\alpha}-Q(x)^{\alpha} \text { for } x \geq x_{0} .
$$

We are going to show that for every $f \in D$ there exists an integer $n_{0}(f)$ such that

$$
\int_{0}^{x_{0}} \frac{1}{n} \sum_{k=1}^{n} P^{k} f(x) d x \geq \frac{\varepsilon}{2 M} \quad \text { for } n \geq n_{0}(f)
$$


where

$$
M:=\sup _{\left[0, x_{0}\right]}\left|(Q(\lambda(x)))^{\alpha}-Q(x)^{\alpha}-\varrho\right| .
$$

Using (2.1) with $W(x)=x^{\alpha}$ and $f \in D$ we have

$$
\begin{aligned}
\int_{0}^{\infty}(Q(\lambda(x)))^{\alpha} P f(x) d x & =\int_{0}^{\infty} f(y) d y \int_{0}^{\infty}(x+Q(y))^{\alpha} h(x) d x \\
& \leq \int_{0}^{\infty} f(y) d y \int_{0}^{\infty}\left(x^{\alpha}+Q(y)^{\alpha}\right) h(x) d x \\
& =\sigma+\int_{0}^{\infty} f(y) Q(y)^{\alpha} d y
\end{aligned}
$$

Fix $f \in D$ such that

$$
\int_{0}^{\infty} Q(x)^{\alpha} f(x) d x<\infty
$$

and define

$$
f_{n}=\frac{1}{n} \sum_{k=1}^{n} P^{k} f \quad \text { for } n=1,2, \ldots
$$

From (2.3), (2.6) and (2.7) it follows that

$$
\int_{0}^{\infty}(Q(\lambda(x)))^{\alpha} P f_{n}(x) d x \leq \sigma+\int_{0}^{\infty} Q(x)^{\alpha} f_{n}(x) d x
$$

and that the integral on the right hand side is finite for every $n$. Hence

$$
\int_{0}^{\infty}\left((Q(\lambda(x)))^{\alpha}-Q(x)^{\alpha}\right) f_{n}(x) d x \leq \sigma+\frac{1}{n} \int_{0}^{\infty}(Q(\lambda(x)))^{\alpha} P f(x) d x .
$$

Since $\sigma<\varrho-\varepsilon$, there exists a positive integer $n_{0}(f)$ such that

$$
\int_{0}^{\infty}\left((Q(\lambda(x)))^{\alpha}-Q(x)^{\alpha}\right) f_{n}(x) d x \leq \varrho-\varepsilon \quad \text { for } n \geq n_{0}(f) .
$$

On the other hand, taking into account (2.3) we have

$$
\begin{aligned}
\int_{0}^{\infty}\left((Q(\lambda(x)))^{\alpha}\right. & \left.-Q(x)^{\alpha}\right) f_{n}(x) d x \\
& \geq \int_{0}^{x_{0}}\left((Q(\lambda(x)))^{\alpha}-Q(x)^{\alpha}\right) f_{n}(x) d x+\varrho \int_{x_{0}}^{\infty} f_{n}(x) d x .
\end{aligned}
$$


Consequently,

$$
\begin{aligned}
\int_{0}^{x_{0}}\left((Q(\lambda(x)))^{\alpha}-Q(x)^{\alpha}\right) f_{n}(x) d x & \leq \varrho-\varepsilon-\varrho \int_{x_{0}}^{\infty} f_{n}(x) d x \\
& =\varrho \int_{0}^{x_{0}} f_{n}(x) d x-\varepsilon
\end{aligned}
$$

for $n \geq n_{0}(f)$, which together with (2.5) gives

$$
-M \int_{0}^{x_{0}} f_{n}(x) d x \leq \int_{0}^{x_{0}}\left((Q(\lambda(x)))^{\alpha}-Q(x)^{\alpha}-\varrho\right) f_{n}(x) d x \leq-\varepsilon
$$

for $n \geq n_{0}(f)$. This implies (2.4) and even a stronger inequality with the right hand side $\varepsilon / M$. The above argument was valid for $f$ satisfying (2.7). To get (2.4) for every $f \in D$ it is enough to observe that the set of all $f \in D$ such that (2.7) holds is dense in $D$.

Now we are going to show that there exists a $\delta>0$ such that

$$
\int_{E} \operatorname{Pf}(x) d x \leq \frac{\varepsilon}{4 M} \quad \text { for } f \in D \text { and } E \subset\left[0, x_{0}\right], m(E) \leq \delta .
$$

In fact, since $h$ is integrable we can find a $\gamma>0$ such that

$$
\int_{F} h(x) d x \leq \frac{\varepsilon}{4 M} \quad \text { for } m(F) \leq \gamma .
$$

Further, since $Q \circ \lambda$ is absolutely continuous, there exists a $\delta>0$ such that

$$
m(Q(\lambda(E))) \leq \gamma \quad \text { for } E \subset\left[0, x_{0}\right], m(E) \leq \delta .
$$

Now let $E \subset\left[0, x_{0}\right]$ be measurable and $m(E) \leq \delta$. Setting $W=1_{Q(\lambda(E))}$ we have $1_{E} \leq W \circ Q \circ \lambda$, which according to (2.1) gives

$$
\begin{aligned}
\int_{E} P f(x) d x & =\int_{0}^{\infty} 1_{E}(x) P f(x) d x \leq \int_{0}^{\infty} W(Q(\lambda(x))) P f(x) d x \\
& =\int_{0}^{\infty} f(y) d y \int_{0}^{\infty} W(x+Q(y)) h(x) d x \\
& =\int_{0}^{\infty} f(y) d y \int_{Q(\lambda(E))-Q(y)} h(x) d x \leq \frac{\varepsilon}{4 M}
\end{aligned}
$$

for every $f \in D$.

In order to verify condition (1.2) fix an $f \in D$ and a positive integer $n \geq n_{0}(f)$ such that (2.4) is satisfied. Fix a measurable set $E \subset\left[0, x_{0}\right]$ with 
$m(E) \leq \delta$. Then

$$
\int_{E} f_{n}(x) d x=\int_{E} P\left(\frac{1}{n} \sum_{k=0}^{n-1} P^{k} f\right) d x \leq \frac{\varepsilon}{4 M}
$$

and using (2.4) we obtain

$$
\begin{aligned}
\int_{\left(x_{0}, \infty\right) \cup E} f_{n}(x) d x & =1-\int_{0}^{x_{0}} f_{n}(x) d x+\int_{E} f_{n}(x) d x \\
& \leq 1-\frac{\varepsilon}{2 M}+\frac{\varepsilon}{4 M}=1-\frac{\varepsilon}{4 M}
\end{aligned}
$$

for $n \geq n_{0}(f)$. Now let $L_{0}$ be a Banach limit and let

$$
L\left(a_{k}\right)=L_{0}\left(\frac{1}{k} \sum_{i=1}^{k} a_{i}\right)
$$

for every bounded sequence $\left(a_{k}\right)$ of real numbers. Evidently, $L$ is also a Banach limit and

$$
L\left(\int_{\left(x_{0}, \infty\right) \cup E} P^{n} f(x) d x\right)=L_{0}\left(\int_{\left(x_{0}, \infty\right) \cup E} f_{n}(x) d x\right) \leq 1-\frac{\varepsilon}{4 M},
$$

which shows that (1.2) with $A=\left[0, x_{0}\right]$ is satisfied.

Now we use Corollary 1.2 to find a sufficient condition for the asymptotic stability of the operator $P$ given by $(0.1),(0.2)$.

THEOREM 2.2. If there exists a positive number $\alpha \leq 1$ such that (2.2) holds, and a nonnegative number $c$ such that

$$
h(x)>0 \quad \text { for a.e. } x \geq c,
$$

then the operator $P$ given by (0.1), (0.2) is asymptotically stable.

Proof. According to Theorem 2.1 the operator $P$ has a stationary density $f_{*}$. Define $C=\operatorname{supp} f_{*}$ and fix positive numbers $\varepsilon, \varrho, x_{0}$ such that $(2.3)$ holds. Further, choose a positive number $a$ such that

$$
\lambda(a) \geq x_{0}, \quad Q(\lambda(a)) \geq c+Q\left(x_{0}\right),
$$

and define

$$
A=\left\{x \geq a:(Q \circ \lambda)^{\prime}(x)>0\right\} .
$$

Since $Q \circ \lambda$ is absolutely continuous and $\lim _{x \rightarrow \infty} Q(\lambda(x))=\infty$, the set $A$ is unbounded (ess $\sup A=\infty)$. Finally, define the number $M$ by (2.5). 
If $x \in A$, then

$$
\begin{aligned}
f_{*}(x) & =P f_{*}(x)=(Q \circ \lambda)^{\prime}(x) \int_{0}^{\lambda(x)} h(Q(\lambda(x))-Q(y)) f_{*}(y) d y \\
& \geq(Q \circ \lambda)^{\prime}(x) \int_{0}^{x_{0}} h(Q(\lambda(x))-Q(y)) f_{*}(y) d y
\end{aligned}
$$

and

$$
(Q \circ \lambda)^{\prime}(x)>0, \quad h(Q(\lambda(x))-Q(y))>0 \quad \text { for } y \in\left[0, x_{0}\right] .
$$

From (2.4) with $f=f_{*}$ it follows that

$$
\int_{0}^{x_{0}} f_{*}(y) d y>0
$$

This shows that $f_{*}(x)>0$ for $x \in A$ and that $A \subset C$. Using (2.4) it is also easy to show that

$$
\sup _{n} \int_{C} P^{n} f(x) d x \geq \frac{\varepsilon}{2 M} \int_{Q(\lambda(a))}^{\infty} h(u) d u \quad \text { for } f \in D .
$$

In fact, according to (2.4) for every density $f$ there is a positive integer $k$ such that

$$
\int_{0}^{x_{0}} P^{k} f(x) d x \geq \frac{\varepsilon}{2 M}
$$

and consequently,

$$
\begin{aligned}
\int_{C} P^{k+1} f(x) d x & \geq \int_{A} P^{k+1} f(x) d x \\
& =\int_{A}(Q \circ \lambda)^{\prime}(x) d x \int_{0}^{\lambda(x)} h(Q(\lambda(x))-Q(y)) P^{k} f(y) d y \\
& \geq \int_{A}(Q \circ \lambda)^{\prime}(x) d x \int_{0}^{x_{0}} h(Q(\lambda(x))-Q(y)) P^{k} f(y) d y \\
& =\int_{0}^{x_{0}} P^{k} f(y) d y \int_{a}^{\infty}(Q \circ \lambda)^{\prime}(x) h(Q(\lambda(x))-Q(y)) d x \\
& \geq \int_{0}^{x_{0}} P^{k} f(y) d y \int_{Q(\lambda(a))}^{\infty} h(u) d u \geq \frac{\varepsilon}{2 M} \int_{Q(\lambda(a))}^{\infty} h(u) d u .
\end{aligned}
$$

Finally, observe that for every density $f$ there exists a positive number 
$b=b(f)$ such that

$$
\operatorname{Pf}(x)>0 \quad \text { for } x \in[b, \infty) \cap A .
$$

To show this choose $b_{0}>0$ such that $\int_{0}^{b_{0}} f(y) d y>0$, and $b>0$ such that $\lambda(b) \geq b_{0}, Q(\lambda(b)) \geq c+Q\left(b_{0}\right)$. For $x \in[b, \infty) \cap A$ we then have

$$
P f(x) \geq(Q \circ \lambda)^{\prime}(x) \int_{0}^{b_{0}} h(Q(\lambda(x))-Q(y)) f(y) d y>0 .
$$

Setting $d=d(f, g)=\max \{b(f), b(g)\}$ we obtain

$$
m(\operatorname{supp} P f \cap \operatorname{supp} P g) \geq m([d, \infty) \cap A)>0 \quad \text { for } f, g \in D .
$$

Thus all the requirements of Corollary 1.2 are satisfied and the proof is complete.

The following example shows that assumption (2.9) in the statement of Theorem 2.2 is essential.

EXAmPle 2.1. Let $h:[0, \infty) \rightarrow[0, \infty)$ be an integrable function such that

$$
\int_{0}^{\infty} h(x) d x=1 \quad \text { and } \quad h(x)=0 \quad \text { for } x \geq \sqrt{c}-c
$$

where $c \in(0,1)$ is a constant. Consider the operator $P: L^{1} \rightarrow L^{1}$ given by the formula

$$
P f(x)= \begin{cases}\frac{1}{2 \sqrt{x}} \int_{0}^{\sqrt{x}} h(\sqrt{x}-y) f(y) d y & \text { for } x \in(0,1) \\ 2 \int_{0}^{2 x-1} h(2 x-y-1) f(y) d y & \text { for } x \geq 1\end{cases}
$$

In this case $Q(x)=x$,

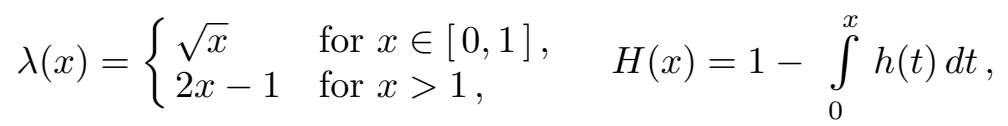

and evidently the assumptions (K1) and (K2) are satisfied. Moreover, for every $\alpha \in(0,1]$,

$$
\int_{0}^{\infty} x^{\alpha} h(x) d x<1<\infty=\lim _{x \rightarrow \infty}\left((Q(\lambda(x)))^{\alpha}-Q(x)^{\alpha}\right) .
$$

According to Theorem 2.1 the operator $P$ has a stationary density. Using (2.11) it is easy to verify the following property of $P$. If $\operatorname{supp} f \subset[1, \infty)$ then supp $\operatorname{Pf} \subset[1, \infty)$ and if $\operatorname{supp} f \subset(0, c)$ then supp $\operatorname{Pf} \subset(0, c)$. Since $c<1$, condition (1.1) cannot be satisfied with an $f_{*}$ independent on $f$. Thus $P$ is not asymptotically stable. 
In the previous results concerning the operator $(0.1),(0.2)$ an important role was played by condition (2.2). Thus a natural question arises: What could we say about the behaviour of $\left(P^{n} f\right)$ when $(2.2)$ is not satisfied? A partial answer to this question may be given by showing that if an opposite condition to $(2.2)$ is satisfied then the operator $P$ is sweeping [2].

We say that a Markov operator $P: L^{1}([0, \infty)) \rightarrow L^{1}([0, \infty))$ is sweeping if

$$
\lim _{n \rightarrow \infty} \int_{0}^{r} P^{n} f(x) d x=0 \quad \text { for every } f \in D \text { and } r \geq 0
$$

Theorem 2.3. Assume that

$$
\sup _{x \geq x_{0}}\left((Q(\lambda(x)))^{\beta}-Q(x)^{\beta}\right)<\int_{0}^{\infty} x^{\beta} h(x) d x<\infty
$$

for an $x_{0} \geq 0$ and $\beta \geq 1$ and that

$$
\int_{Q\left(\lambda\left(x_{0}\right)\right)}^{\infty} h(x) d x>0 .
$$

Then the operator $P$ given by $(0.1),(0.2)$ is sweeping.

Pr o of. Define

$$
z_{0}=\left(Q\left(\lambda\left(x_{0}\right)\right)\right)^{\beta}, \quad w(z)= \begin{cases}e^{-\varepsilon z_{0}} & \text { for } z \in\left[0, z_{0}\right] \\ e^{-\varepsilon z} & \text { for } z>z_{0}\end{cases}
$$

and

$$
V(x)=w\left((Q(\lambda(x)))^{\beta}\right)
$$

where $\varepsilon>0$ will be chosen later. We shall show that there exists a nonnegative constant $\gamma<1$ such that

$$
\int_{0}^{\infty} V(x) P f(x) d x \leq \gamma \int_{0}^{\infty} V(x) f(x) d x \quad \text { for } f \in D .
$$

Since $V(x)$ admits a positive minimum on every compact set this inequality implies (2.12) (see also [2]).

According to (2.13) there exists a number $\varrho$ such that

$$
\sup _{x \geq x_{0}}\left((Q(\lambda(x)))^{\beta}-Q(x)^{\beta}\right)<\varrho<\int_{0}^{\infty} x^{\beta} h(x) d x .
$$

Define

$$
I(y)=\int_{0}^{\infty} \frac{w\left((x+Q(y))^{\beta}\right)}{V(y)} h(x) d x \quad \text { for } y \geq 0 .
$$


If $y \leq x_{0}$, then $V(y)=w\left(z_{0}\right)$ and

$$
\begin{aligned}
I(y) & \leq \int_{0}^{\infty} \frac{w\left(x^{\beta}\right)}{V(y)} h(x) d x=\int_{0}^{Q\left(\lambda\left(x_{0}\right)\right)} \frac{w\left(x^{\beta}\right)}{w\left(z_{0}\right)} h(x) d x+\int_{Q\left(\lambda\left(x_{0}\right)\right)}^{\infty} \frac{w\left(x^{\beta}\right)}{w\left(z_{0}\right)} h(x) d x \\
& =\int_{0}^{Q\left(\lambda\left(x_{0}\right)\right)} h(x) d x+\int_{Q\left(\lambda\left(x_{0}\right)\right)}^{\infty} h(x) e^{-\varepsilon\left(x^{\beta}-z_{0}\right)} d x \\
& =1-\int_{Q\left(\lambda\left(x_{0}\right)\right)}^{\infty} h(x)\left(1-e^{-\varepsilon\left(x^{\beta}-z_{0}\right)}\right) d x=: \gamma_{1}(\varepsilon)<1 .
\end{aligned}
$$

If $y>x_{0}$, then $(Q(\lambda(y)))^{\beta}-Q(y)^{\beta}<\varrho$ and, since $w(z) \leq e^{-\varepsilon z}$ for $z \geq 0$,

$$
\frac{w\left((x+Q(y))^{\beta}\right)}{V(y)} \leq \frac{e^{-\varepsilon(x+Q(y))^{\beta}}}{e^{-\varepsilon(Q(\lambda(y)))^{\beta}}} \leq \frac{e^{-\varepsilon(x+Q(y))^{\beta}}}{e^{-\varepsilon\left(\varrho+Q(y)^{\beta}\right)}} \leq e^{-\varepsilon\left(x^{\beta}-\varrho\right)} ;
$$

consequently,

$$
I(y) \leq \int_{0}^{\infty} h(x) e^{-\varepsilon\left(x^{\beta}-\varrho\right)} d x=: \gamma_{2}(\varepsilon) .
$$

From Lemma 2.1 it follows that

$$
\begin{aligned}
\int_{0}^{\infty} V(x) P f(x) d x & =\int_{0}^{\infty} f(y) d y \int_{0}^{\infty} w\left((x+Q(y))^{\beta}\right) h(x) d x \\
& =\int_{0}^{\infty} f(y) V(y) I(y) d y \\
& \leq \gamma_{1}(\varepsilon) \int_{0}^{x_{0}} V(y) f(y) d y+\gamma_{2}(\varepsilon) \int_{x_{0}}^{\infty} V(y) f(y) d y
\end{aligned}
$$

for every density $f$. Since $\gamma_{1}(\varepsilon)<1$ for every $\varepsilon>0$, in order to show (2.14) with a constant $\gamma<1$ it is enough to prove that there exists and $\varepsilon>0$ such that $\gamma_{2}(\varepsilon)<1$. But the function $\gamma_{2}$ is differentiable on $[0, \infty)$ and

$$
\gamma_{2}^{\prime}(\varepsilon)=-\int_{0}^{\infty} h(x)\left(x^{\beta}-\varrho\right) e^{-\varepsilon\left(x^{\beta}-\varrho\right)} d x
$$

whence

$$
\gamma_{2}^{\prime}(0)=\varrho-\int_{0}^{\infty} x^{\beta} h(x) d x<0 .
$$

Consequently, for sufficiently small $\varepsilon>0$ we have $\gamma_{2}(\varepsilon)<\gamma_{2}(0)=1$, which completes the proof. 


\section{References}

[1] S. R. Foguel, The Ergodic Theory of Markov Processes, Van Nostrand Math. Stud. 21, Van Nostrand, 1969.

[2] H. Gacki and A. Lasota, Markov operators defined by Volterra type integrals with advanced argument, Ann. Polon. Math. 51 (1990), 155-166.

[3] T. Komorowski and J. Tyrcha, Asymptotic properties of some Markov operators, Bull. Polish Acad. Sci. Math. 37 (1989), 221-228.

[4] U. Krengel, Ergodic Theorems, de Gruyter, 1985.

[5] A. Lasota and M. C. Mackey, Globally asymptotic properties of proliferating cell populations, J. Math. Biol. 19 (1984), 43-62.

[6] - - -, Probabilistic Properties of Deterministic Systems, Cambridge University Press, 1985.

[7] A. Lasota, M. C. Mackey and J. Tyrcha, The statistical dynamics of recurrent biological events, J. Math. Biol. 30 (1992), 775-800.

[8] J. Malczak, An application of Markov operators in differential and integral equations, Rend. Sem. Mat. Univ. Padova, in press.

[9] J. Socała, On the existence of invariant densities for Markov operators, Ann. Polon. Math. 48 (1988), 51-56.

[10] J. Tyrcha, Asymptotic stability in a generalized probabilistic/deterministic model of the cell cycle, J. Math. Biol. 26 (1988), 465-475.

[11] J. J. Tyson and K. B. Hannsgen, Global asymptotic stability of the size distribution in probabilistic model of the cell cycle, J. Math. Biol. 22 (1985), 61-68.

[12] -, -, Cell growth and division: A deterministic/probabilistic model of the cell cycle, ibid. 23 (1986), 231-246.

INSTITUTE OF MATHEMATICS

SILESIAN UNIVERSITY

BANKOWA 14

40-007 KATOWICE, POLAND 